\title{
Defining modifiable barriers to uptake of dietary recommendations in Chinese immigrants with type 2 diabetes: a qualitative study
}

\author{
Feiyue Deng $^{\mathrm{a}}$ and Catherine B. Chan ${ }^{\mathrm{ab} \star}$ \\ ${ }^{a}$ Department of Agricultural, Food and Nutritional Science, University of Alberta, Edmonton, AB T6G \\ 2R3, Canada; ${ }^{b}$ Department of Physiology, University of Alberta, Edmonton, AB T6G 2H7, Canada \\ *cbchan@ualberta.ca
}

\begin{abstract}
The objective is to support development of a nutritional intervention for Chinese immigrants with diabetes by defining current deficiencies and identifying modifiable factors and mechanisms of change. Semi-structured interviews conducted with 13 ethnic Chinese with type 2 diabetes identified modifiable problems related to culturally relevant diabetes resources and low cultural acceptability of recommended diets. These factors could be addressed through creation of resources developed in partnership with Chinese-speaking health care professionals and persons with diabetes.
\end{abstract}

Key words: acculturation, food acceptability, dietary adherence, Chinese immigrants, type 2 diabetes, intervention development

\section{OPEN ACCESS}

Citation: Deng F and Chan CB. 2019. Defining modifiable barriers to uptake of dietary recommendations in Chinese immigrants with type 2 diabetes: a qualitative study. FACETS 4: 55 I-565. do: 10.1139/ facets-20 | 9-000 |

Handling Editor: Charles Couillard

Received: January 8, 2019

Accepted: July 10, 2019

Published: October 28, 2019

Copyright: (c) 2019 Deng and Chan. This work is licensed under a Creative Commons Attribution 4.0 International License (CC BY 4.0), which permits unrestricted use, distribution, and reproduction in any medium, provided the original author(s) and source are credited.

Published by: Canadian Science Publishing

\section{Introduction}

Immigrants such as Chinese-Americans are more prone to diabetes than their peers living in China (Xu et al. 2011), and Chinese are identified as higher risk than some other ethnic groups in Canada (Alangh et al. 2013). While immigrants are screened and thus generally healthy (i.e., "healthy immigrant effect") when arriving in a new country (Sanou et al. 2014), they subsequently experience adaptation to a new environment and culture, termed "acculturation" (Deng et al. 2013) and besides nutrition face challenges including language, social norms, and shift in social status that contributes to development of obesity and chronic diseases (Perez 2002; Lear et al. 2009).

In Canada, $\sim 15 \%$ of Chinese immigrants cannot speak English or French (Chui et al. 2005), similar to American Chinese populations, which also report lower acculturation and English language proficiency (Kandula et al. 2008). The tendency of Chinese in Canada to live in ethnic enclaves in metropolitan areas (Chui et al. 2005; Walks and Bourne 2006; Statistics Canada 2012) may reduce impetus to acculturate more fully. While this lack of acculturation might be predicted to protect new immigrants from poor diet and chronic diseases, the data are mixed. A survey of 106 middle-aged Chinese Canadians, most of whom had lived in Canada $>10$ years, found that $98 \%$ continued to consume a traditional Chinese dinner, whereas two-thirds ate a western-style breakfast (Kwok et al. 2009); however, the health of the respondents was not described. Dietary improvements of Chinese living in Canada included higher vegetable and fruit consumption and reduced consumption of 
deep-fried foods, whereas portion sizes, dining out, and consumption of convenience foods increased (Rosenmoller et al. 2011). Immigrants to Canada who were born in China were more than twice as likely to have inadequate intake of protein, iron, calcium, and several vitamins as Canadian-born residents (Pomerleau et al. 2005).

Acculturation can increase disease risk because immigrants tend to increase their consumption of fats, sweets, and soft drinks (Pan et al. 1999; Lv and Cason 2004), which then increases risk of obesity and cardiovascular disease (Beasley et al. 2018). Increased consumption of western and reduced consumption of traditional Chinese foods was commonly reported by Chinese immigrants to the United States (Lv and Cason 2004) necessitated by low availability of affordable traditional Chinese foods (Satia et al. 2000). Intake of processed, ready-to-eat western foods may be favoured due to low familiarity with western cooking methods (Lv and Brown 2010).

Chinese retain their traditional diet more faithfully and for a longer time after immigration than other groups (Tan 2011). Low acculturation can impede disease treatment and management. For example, lack of English fluency limits access to diabetes-related knowledge, which attenuates patients' capacity for diabetes self-management (Anderson et al. 1995). Following a prescribed diet for chronic diseases such as type 2 diabetes (T2D) is complicated by ethnocultural influences on dietary behaviours (Franzen and Smith 2009). Responsiveness to factors such as food acceptability, which involves both sensory and cultural factors, may improve dietary adherence (Coyne et al. 1995, Jimenez-Cruz et al. 2003). For Chinese-Americans with T2D, culturally tailored self-management programs effectively sustained participation and improved diabetes knowledge and glycemic control (Deng et al. 2018). However, detailed information identifying the problems, modifiable factors and potential solutions has not been published for these programs despite being essential parts of the process of intervention development (Wight et al. 2016).

As first steps in developing a culturally relevant nutrition intervention for Chinese immigrants in Canada, we sought to address two main questions: (i) What resources are available for Chinese immigrants to help them manage diabetes through diet? (ii) What are the barriers and facilitators for Chinese immigrants to adhering to current recommendations? To address these questions, the Conceptual Model of Diabetes Self-management among Chinese Immigrants, based on an extensive literature review on studies conducted among Chinese immigrants in the US (Zeng et al. 2014), was adopted. This framework identified five categories of factors that may impact diabetes self-management in Chinese immigrants: socio-demographic characteristics, behavioral and psychological characteristics, social support, linguistics barriers, and cultural characteristics.

\section{Methods}

\section{Participants}

The study was approved by the University of Alberta Health Research Ethics Board (Pro00023449). Participants were recruited through advertisements in hospitals, Chinese supermarkets, cultural centres, and newspapers in Edmonton, Alberta. Prospective participants were screened against the inclusion criteria via telephone: Chinese descent, $\geq 35$ years of age, diagnosed with T2D or prediabetes, living in North America $\geq 1$ year, read and write English or Chinese. Those meeting the criteria $(n=14)$ provided written informed consent. Consented participants received a $\$ 25$ gift card for a local grocery store. For the purposes of this analysis, persons with prediabetes $(n=1)$ were omitted because of potential confounding by differences in treatment intensity compared with persons with diabetes, thus responses from $n=13$ participants were included. 


\section{Study design and implementation}

A qualitative study employing one-on-one interviews was conducted. The bilingual (Mandarin, English) study coordinator held two meetings at the University of Alberta with each participant. At the first meeting, hemoglobin A1C (a marker of blood glucose control) and anthropometric measures were taken (Asaad et al. 2016). Demographic information was collected through a questionnaire. Perceived dietary adherence was evaluated by a questionnaire asking how often in the previous week (days out of $7 \mathrm{~d}$ ) people had followed each of nine recommendations from Diabetes Canada Nutrition Therapy Guidelines, with a maximum score of 63 for high adherence (Asaad et al. 2015). The actual dietary adherence was reflected by participants' Healthy Eating Index (HEI) scores. The HEI scoring criteria were adapted from the American Healthy Eating Index scoring criteria, and followed the recommendations from Canada's Food Guide (Garriguet 2009). The total HEI score was calculated and could range from 0 (low diet quality) to 100 (high diet quality). Physical activity was assessed by the Godin-Shephard Leisure-Time Physical Activity Questionnaire, which acquired the frequency of strenuous, moderate, and mild physical activities. Scores for total physical activity were calculated (Godin 2011). Acculturation was assessed by the Suinn-Lew Asian Selfidentity Acculturation Scale, which is a validated scale for the assessment of acculturation among Asian-Americans (Suinn et al. 1992). The adaptation was that the word Asian was replaced with Chinese, so that it was more specific to Chinese participants. It consisted of 21 questions inquiring about various aspects of culture including food preferences. A score was obtained through adding up the answers for all 21 questions and then dividing the total value by 21 . Scores for individual items could range from 1 (low acculturation) to 5 (high acculturation). All questionnaires were available in both English and Chinese with accuracy verified by back-translation to English. Descriptive data were expressed as means \pm SD or proportions.

During the second meeting, a one-on-one, 40-minute, semi-structured interview was conducted, focusing on facilitators and barriers to following current nutritional guidelines, health beliefs, factors influencing food choices, and opinions on the development of a culturally relevant menu plan for Chinese diabetes patients. The questions comprising the interview (Supplementary Material S1) proceeded in English $(n=3)$ or Mandarin $(n=11)$ by one researcher/author (FD) and were recorded using a digital voice recorder.

\section{Qualitative analysis}

An audit trail protocol was developed to ensure transferability, i.e., the extent to which the findings from this study could be generalized to similar situations (Merriam 2002). Interviews were transcribed verbatim by the same researcher (FD). The protocol included the researcher (FD) reading the transcripts multiple times to ensure a comprehensive understanding prior to coding. Transcriptions were coded manually in Microsoft Word using an iterative approach where the researcher went back repeatedly to add or modify the codes until the information was properly captured. A research assistant (AZ) who was also fluent in Mandarin double-checked the codes against the transcripts. The researcher summarized the interview and confirmed the main points using a process called member checking to allow the participant to add anything missed or correct any misinterpretation. The preliminary analysis including codes was communicated back to the participants via email or phone call to ask for their views and evaluation of veracity. The goal of this step was to reduce bias introduced by the researcher (Creswell 2007) and increase credibility of the findings (Marshall and Rossman 2006). After member checking, codes generated from all transcripts were translated into English, pooled and organized in categories aligned with the research questions. Themes were generated from each category to answer the research questions, which was done in a similar iterative way as the coding process (FD); this was verified (CBC) with discrepancies discussed and resolved. 
Table 1. Participant demographics and heath characteristics $(n=13)$.

Characteristics

Age (year)

Age at immigration (year)

Years in North America

Household annual income (C\$) $(n=12)$

Household annual income per capita (C\$) $(n=12)$

A1C (\%)

Duration of diabetes (year)

BMI $\left(\mathrm{kg} / \mathrm{m}^{2}\right)$

Waist circumference $(\mathrm{cm})$, females $(n=8)$

Waist circumference $(\mathrm{cm})$, males $(n=5)$

Central obesity (female $>86 \mathrm{~cm}$, male $>90 \mathrm{~cm}$ )

Healthy Eating Index Score (maximum 100)

Perceived Dietary Adherence Score (maximum 63)

Leisure-time physical activity score (no maximum)

Acculturation score (minimum 1, maximum 5)

Education

Less than high school

High school

College diploma

University degree

Post-graduate degree

Employment

Retirement income

Wages and salaries

No income (receive money from or live with children)

Place of birth

Mainland China

Taiwan

Hong Kong

Southeast Asia

Canada
Mean \pm SD

$63 \pm 15$

$38 \pm 20$

$25 \pm 17$

$37000 \pm 19479$

$18069 \pm 7979$

$6.9 \pm 1.3$

$7.5 \pm 7.8$

$24.3 \pm 2.8$

$86.5 \pm 8.3$

$95.7 \pm 10.5$

$53.8 \%$

$66.3 \pm 9.8$

$39.7 \pm 7.6$

$25.0 \pm 17.6$

$1.89 \pm 0.43$

Percent

36

7

36

7

14

50

36

14

50

14

21

0

\section{Results and discussion}

\section{Demographic, health, acculturation and dietary characteristics}

Table 1 summarizes participant characteristics. Average income fell below the low-income cut-off for a census metropolitan area (Income Statistics Division 2015). The majority had normal weight, none 
was obese, but $64.3 \%$ had central obesity. Acculturation was low ( $<2$ out of 5$)$. Diet quality measured by the Healthy Eating Index (Garriguet 2009) (mean = 66 out of 100, categorized as "acceptable") is similar (mean $=69)$ to a mainly Caucasian cohort (Asaad et al. 2016). Adherence to 2013 Diabetes Canada Nutrition Therapy Guidelines measured by the Perceived Dietary Adherence Questionnaire (mean $=40$ out of 63 ) was somewhat higher than two mainly Caucasian groups (both mean $=32$ ) (Asaad et al. 2016; Raj et al. 2018). The leisure-time physical activity score of 25 is interpreted as "active" on the Godin Scale (Godin 2011).

\section{Defining and understanding the problem and its causes}

The objective was to explore factors that influenced food choice, diet quality, and barriers to acquiring nutrition knowledge to identify specific problems as the first step in quality intervention development (Wight et al. 2016), i.e., defining and understanding the problem and its causes, identifying which causal or contextual factors are modifiable, and deciding on the mechanisms of change. This objective was addressed by analyzing participant responses to questions based on general concepts presented in the Conceptual Model of Diabetes Self-management among Chinese Immigrants (Zeng et al. 2014). The model is similar to more generalized discussions of social factors that have an impact on diabetes self-management, including demographic, social, psychological, and economic factors in immigrants (Gonzalez-Zacarias et al. 2016). The discussion points and quotations upon which the interpretations are based are summarized in Table 2 and categorized as barriers or facilitators to dietary adherence, also illustrated in Fig. 1. Individual participants are identified by a study code for Dietary needs Assessment of Chinese with type 2 diabetes [DAC\#].

A main barrier or problem identified was a lack of culturally relevant, practical information for Chinese immigrants with diabetes, thereby lowering the utility of current dietary recommendations. This aligned with cultural characteristics in the conceptual model (Zeng et al. 2014) but also touched on social support because the respondents addressed who they received information from, including health care professionals and community as well as family and friends. When immigrants categorize their patient-provider relationship as poor, they are $16 \%$ less likely to participate in self-management practices (Hyman et al. 2017). Outside of the formal healthcare system [which was cited by participant DAC08], sources of dietary information included the Chinese Community Center [DAC13, DAC09], family and friends [DAC01], and newspapers and the internet [DAC05, DAC07]. Activities at the Chinese community centre including seminars given by a Chinese dietician or by non-Chinese health professionals (translated by a bilingual staff member) were the only sources of culturally relevant dietary information for many participants. Some participants were unaware of existing resources such as the Diabetes Canada website, where there is diabetes management information written in Chinese. Eating Well with Canada's Food Guide (CFG) in Chinese was the main resource cited but only used by a few. Participants with low computer skills noted they could not access it easily from the Diabetes Canada website. Some participants had only received general recommendations from their family doctors, such as to reduce sugar and fat intake and to lose weight without further guidance [DAC13], and one took action based on "common sense" [DAC12]. Another helpful recommendation was about portion control [DAC04] but others found portion information confusing [DAC13].

Language was a barrier, especially among the elderly who tended to have lower English-language proficiency. The participants who mentioned language ranged in age from 68 to 75 years. Only one participant $>70$ years of age did not mention language. Moreover, for these participants, the language proficiency scale within the acculturation instrument averaged 1.33 versus 2.00 for the whole cohort. One participant did not attend the diabetes education program because she could not speak English and her English-speaking daughter was unavailable to accompany her. Another unilingual participant did not read any dietary information because it was mostly in English. These findings are consistent 
Table 2. Documentation of participants responses to the research questions categorized according to the conceptual model, theme and barrier/facilitator.

\begin{tabular}{|c|c|c|c|c|c|}
\hline Research question & $\begin{array}{c}\text { Conceptual model } \\
\text { category }^{a}\end{array}$ & $\begin{array}{l}\text { Emergent } \\
\text { themes }\end{array}$ & $\begin{array}{l}\text { Barrier or } \\
\text { facilitator }\end{array}$ & $\begin{array}{l}\text { Participant } \\
\text { ID }\end{array}$ & Quotations \\
\hline $\begin{array}{l}\text { What resources } \\
\text { are available } \\
\text { for Chinese immigrants } \\
\text { to help them manage } \\
\text { diabetes through diet? }\end{array}$ & Social support & $\begin{array}{l}\text { Information } \\
\text { about } \\
\text { portions } \\
(n=6)\end{array}$ & Facilitator & DAC04 & $\begin{array}{l}\text { "... at least they show me the big difference between you eating } \\
\text { the same amount of food in one portion, and the same amount of } \\
\text { food you divided into small portions.... Before, I had } \\
\text { misunderstood that. The same amount of food, I eat it all at the } \\
\text { same time and once a day, that's what I did before. But after I went } \\
\text { to the class, I learned that there was big difference. I shouldn't } \\
\text { have a big portion, even though it's the same amount of food." }\end{array}$ \\
\hline
\end{tabular}

$\begin{array}{llll}\text { Cultural } & \text { DAC12 } & \text { Barrier } \\ \text { characteristics } & \begin{array}{l}\text { Cultural } \\ \text { relevance } \\ (n=6)\end{array} & \text { DAC04 }\end{array}$

$\begin{array}{lllll} & \begin{array}{l}\text { Linguistic barriers; } \\ \text { sociodemographic } \\ \text { barriers (age) }\end{array} & \begin{array}{l}\text { Language } \\ (n=6)\end{array} & \text { Barrier } & \text { DAC12 } \\ & \text { Not applicable } & \begin{array}{l}\text { Media } \\ \text { (printed or } \\ \text { online) } \\ (n=5)\end{array} & \begin{array}{l}\text { Facilitator and } \\ \text { (or) barrier }\end{array} & \text { DAC05 } \\ \begin{array}{l}\text { Where do Chinese } \\ \text { immigrants get } \\ \text { diabetes-related }\end{array} & & & \\ \text { information? } & & & \end{array}$

$(n=5)$

DAC07 "I got the information from newspaper. They say you should exercise more, and eat more vegetables, but I don't think the information is comprehensive."

DAC13 "I got the information from the Chinese Community Center. They told me to eat more fish, more vegetables, And they gave me a food guide. Tomatoes, grapes, and vegetables, etc., they told me to eat all those foods......"

DAC09 "There are often seminars (about nutrition) at the Chinese Community Center. I go there whenever I have time, and I try to follow their instructions when possible ...... There seems to be a Canada's Food Guide, which tells you how many portions of meat and vegetables you should eat, but our lifestyle is different after all, they like to eat meat......"

Social support
Health care Facilitator and professionals (or) barrier $(n=4)$
DAC08 "I saw a dietitian about 8 years ago, and they showed the guideline from the Canada government. But you know, once you, you attended it, sometimes you forget about it....."

DAC13 "My doctor told me to exercise more, take medications, and eat less sugar... he is Chinese... he didn't give me much information about nutrition..." 
Table 2. (concluded)

\begin{tabular}{|c|c|c|c|c|c|}
\hline Research question & $\begin{array}{l}\text { Conceptual model } \\
\text { category }^{a}\end{array}$ & $\begin{array}{l}\text { Emergent } \\
\text { themes }\end{array}$ & $\begin{array}{l}\text { Barrier or } \\
\text { facilitator }\end{array}$ & $\begin{array}{l}\text { Participant } \\
\quad \text { ID }\end{array}$ & Quotations \\
\hline & & $\begin{array}{l}\text { Family and } \\
\text { friends } \\
(n=3)\end{array}$ & Facilitator & DAC01 & $\begin{array}{l}\text { "My friends would also remind me not to eat too much fat. For } \\
\text { example, when I eat braised eggplant (a traditional Chinese } \\
\text { dish cooked with a lot of oil), they would say, you should avoid } \\
\text { that, it's not good for you." }\end{array}$ \\
\hline \multirow{11}{*}{$\begin{array}{l}\text { What are the barriers } \\
\text { and facilitators for } \\
\text { Chinese immigrants to } \\
\text { adhering to current } \\
\text { recommendations? }\end{array}$} & $\begin{array}{l}\text { Cultural } \\
\text { characteristics }\end{array}$ & $\begin{array}{l}\text { Personal } \\
\text { choice/taste } \\
(n=4)\end{array}$ & Barrier & DAC04 & $\begin{array}{l}\text { “... and personal preference, that is, for example, if they } \\
\text { recommended some food which I don't like, I won't eat it but } \\
\text { will try to find an alternative... " }\end{array}$ \\
\hline & & & & DAC08 & $\begin{array}{l}\text { "... I didn't follow it at all. I just know the basic. I just eat what } \\
\text { I want." }\end{array}$ \\
\hline & $\begin{array}{l}\text { Socio- } \\
\text { demographic } \\
\text { characteristics }\end{array}$ & $\begin{array}{l}\text { Price and } \\
\text { thrift }(n=4)\end{array}$ & Barrier & DAC09 & $\begin{array}{l}\text { "... My wife and I just go to the market, buy whatever is } \\
\text { available, cheap and appropriate for us. We didn't put much } \\
\text { effort into meal planning." }\end{array}$ \\
\hline & Not applicable & $\begin{array}{l}\text { Nutritional } \\
\text { profile }(n=8)\end{array}$ & Facilitator & DAC10 & $\begin{array}{l}\text { "Now when I buy food, I always read the label. If there is a lot of } \\
\text { sugar in it, I won't buy it." }\end{array}$ \\
\hline & $\begin{array}{l}\text { Behavioural and } \\
\text { psychological } \\
\text { characteristics } \\
\text { (health worries) }\end{array}$ & $\begin{array}{l}\text { Health } \\
\text { concerns } \\
(n=7)\end{array}$ & Facilitator & DAC13 & $\begin{array}{l}\text { "Just my diabetes. Diabetes affects my food choices. Without } \\
\text { diabetes, I'd be able to eat anything. It wouldn't matter if I ate a } \\
\text { little more or a little less..." }\end{array}$ \\
\hline & & & & DAC05 & $\begin{array}{l}\text { “... It's like my sister, she has diabetes... One time she fell } \\
\text { down, and then after that, she had to use wheelchair for a few } \\
\text { years. So I don't want that, you know." }\end{array}$ \\
\hline & Not applicable & Time $(n=4)$ & Barrier & DAC05 & $\begin{array}{l}\text { "...you would think that you would have more time to spend } \\
\text { after you get retired, but I feel I still don't have enough time, } \\
\text { and that is probably my biggest barrier (to healthy eating)..." }\end{array}$ \\
\hline & Cultural characteristics & $\begin{array}{l}\text { Eating away } \\
\text { from home } \\
(n=8)\end{array}$ & Barrier & DAC01 & $\begin{array}{l}\text { "... like, my co-workers or friends, they have birthday parties } \\
\text { or things like that, and I would eat a lot of cakes and drink a lot } \\
\text { of beers before I even realize it. Sometimes I get together with } \\
\text { friends, and I would easily eat a lot at a BBQ, such as chicken } \\
\text { wings, burgers or hotdogs. I could easily eat a lot of these foods } \\
\text { if I was not careful." }\end{array}$ \\
\hline & Social support & $\begin{array}{l}\text { Family and } \\
\text { friends } \\
(n=4)\end{array}$ & Facilitator & DAC08 & $\begin{array}{l}\text { "My wife has tried to help me a lot. But the food that she made, } \\
\text { sometimes I don't like it. But mostly it's on the nutrition side, } \\
\text { like all the beans, and all kinds of rice, brown rice..." }\end{array}$ \\
\hline & & & & DAC05 & $\begin{array}{l}\text { "My wife tells me to control sugar. She tells me not to eat too } \\
\text { much stuff that has sugar in it... Like sometimes, when we go out } \\
\text { for dinner, there is dessert right? And she is like, don't eat that!" }\end{array}$ \\
\hline & & & & $\mathrm{DAC} 02$ & $\begin{array}{l}\text { "My wife cooks for me, so I eat whatever she cooks....... she } \\
\text { usually picks food with low GI (glycemic index)..." }\end{array}$ \\
\hline
\end{tabular}

a Zeng et al. (2014).

with the conceptual framework, which identified linguistic barriers as a main contributing factor to diabetes self-management uptake (Zeng et al. 2014). Low language proficiency has been identified as an independent risk factor for poor glycemic control. They also demonstrate that age (a sociodemographic factor identified in the conceptual model) is intertwined with factors such as literacy 


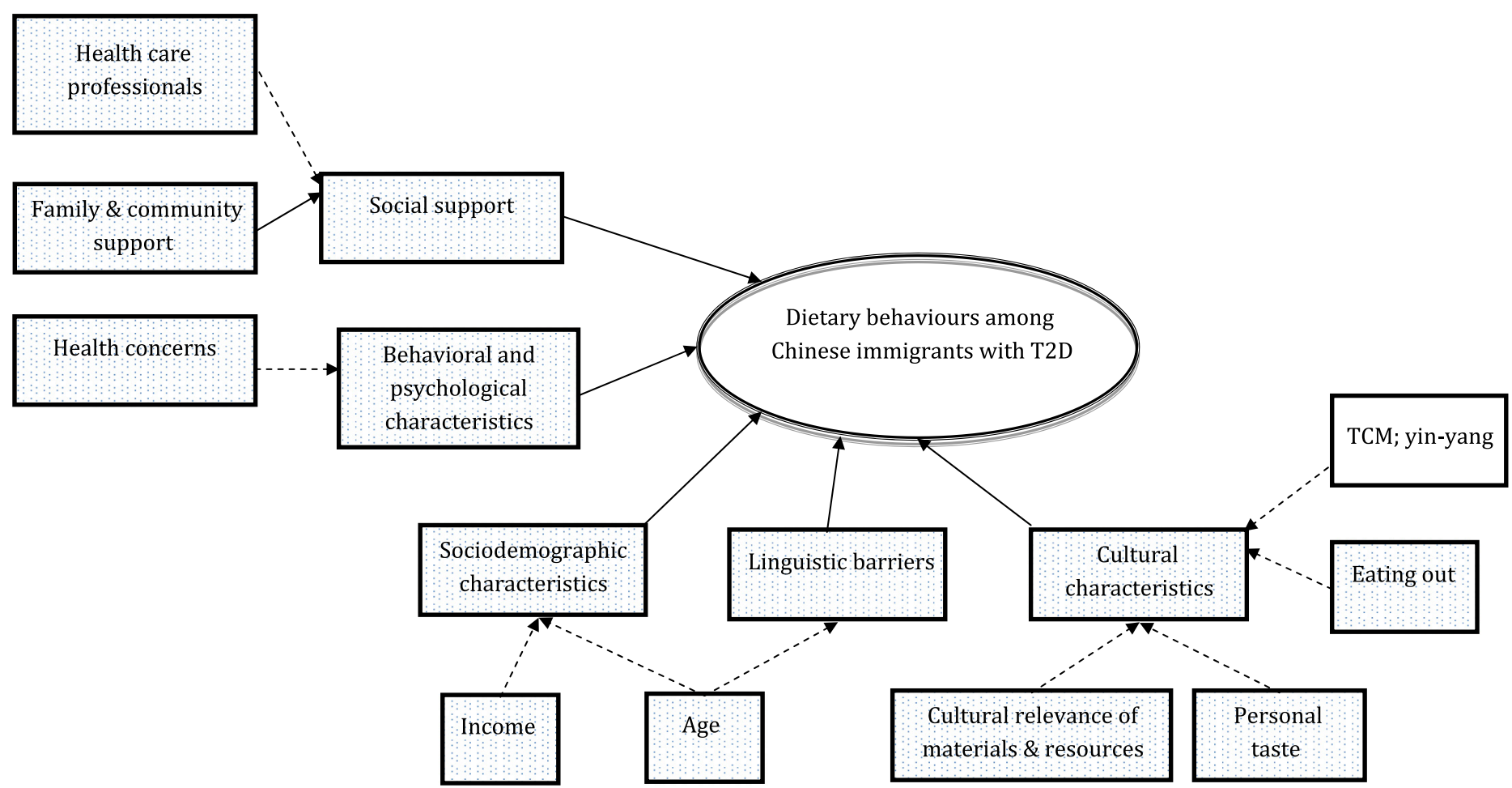

Fig. 1. The conceptual model of Zeng et al. (2014) modified to incorporate themes from the current research. Stippled boxes-themes and concepts identified in the current study; clear box — theme not identified in the current study. Solid lines—facilitators; dashed lines—barriers. Zeng et al. (2014) was distributed under the Creative Commons Attribution License (CC BY 3.0).

and overall acculturation. Low literacy may also include low health literacy as reported in a study of Irish immigrants (Thabit et al. 2009).

Participants repeatedly indicated that the current dietary recommendations lacked consideration of Chinese culture and the dietary habits of Chinese immigrants. Some participants indicated that current recommendations were too general and lacked detailed instructions on meal planning whereas others said that CFG was too complicated and confusing, making it too much trouble to follow. Moreover, the literally translated CFG did not take into account the cultural background and dietary patterns of Chinese immigrants [DAC04]. As a result, many participants thought the information was not helpful and became frustrated when they had to give up some traditional foods. However, rather than a disadvantage of the content of recommendations, this seems more related to their delivery. Misunderstanding of the recommendations could be due to the literacy level of the client (Anderson et al. 1995) as well as how the education was delivered. The conceptual framework cited traditional Chinese health beliefs, such as yin-yang balance and traditional Chinese medicine in immigrant populations but participants in our interviews rarely mentioned these as drivers of health behaviours, as evidenced by a lack of quotations (Table 2). Those who did comment said they paid attention to dietary balance in general but did not seem to consider this as one of the determining factors in their food choices, and others said they did not put much thought into yin-yang balance.

Personal taste preference was an important factor influencing food choices among many participants [DAC04, DAC08]. Taste preferences develop early in an individual's life and thus the cultural 
background and traditional cuisine are the best predictors (Contento 2011). Participants were torn between giving up familiar foods or continuing to eat them while worrying about their effects on glycemic control and fear of complications. Eating out, such as eating at parties and family get-togethers and eating during traveling was another consistently mentioned barrier [DAC10]. Overeating and eating unhealthy food were common when eating out. This is consistent with previous literature on Chinese immigrants as well as other populations (Lv and Cason 2004; Cao et al. 2014; Kwon and Ju 2014; Moreira et al. 2015). Family members could be supportive in this regard, reminding participants to be careful of their diet [DAC05] or preparing healthful meals [DAC02, DAC08], illustrating the importance of social support.

Food price was another important factor as explained by participant [DAC09]; most participants in this study were low income, a sociodemographic factor in the conceptual model (Zeng et al. 2014). Financial stress is a strong predictor of poor diabetes self-management practices in recent immigrants from Asian and South Asian regions (Hyman et al. 2017). Even for one participant with higher income, price was an important concern because he was used to being thrifty [DAC14]. Nutrition and health concerns also played a role in determining food choices; participants suggested that traditional Chinese recipes be modified to contain less fat and salt so that they could enjoy traditional food while being healthy [DAC08, DAC13].

Other barriers were common across cultural boundaries. Some participants, especially those who were working or had family to care for, lacked time to spend on translating guidelines into practice. One participant [DAC02] was hoping for the invention of diabetic foods that could be easily stored and reheated to save time. Another [DAC05] prioritized other activities more highly than following dietary recommendations. Other barriers included lack of willpower and adequate dietary knowledge and cravings. Additional factors mentioned by participants included food quality and variety and convenience related to food shopping.

\section{Identification of modifiable factors and mechanisms of change}

From the common barriers identified by participants, a problem list was developed as summarized in Table 3. From this, modifiable factors were extracted that could be summarized as providing culturally relevant resources for both healthcare professionals and Chinese immigrants with diabetes that would target specific nutritional issues while addressing food acceptability, which includes the types, taste, and price of foods.

Table 3. Defining the problems, identifying modifiable factors and proposing mechanisms of change.

Defining the problem

Identifying modifiable factors

Mechanisms of change

Low acceptability (ethnocultural and hedonic) of recommended diet/foods; unknown healthy properties of traditional foods

Price of food, thriftiness, low income

Limited culturally relevant and practical nutritional information, recommendations and education; limited Chinese-language resources and education

Access to resources
Identify culturally relevant recipes, foods, substitutions

Food selection to minimize cost

Resources for people with diabetes and for health care professionals

Resources for people with diabetes
Culturally relevant food guide; menu plan; specific foods, e.g., calcium-fortified soy products instead of dairy, brown instead of white rice, low-sodium condiments; skill-building (food labels, substitutions)

Practical, flexible menu plan; use of left-overs; shopping support

Co-design resources with patients and healthcare professionals with knowledge of Chinese culture

Create print and online resources 
Mechanisms to address barriers to consuming recommended diets for diabetes in other populations have included such resources as menu plans, grocery lists, and recipes (Cunningham et al. 2006; Bader et al. 2013; Soria-Contreras et al. 2014). Such strategies could illustrate nutritionally appropriate and culturally relevant foods, provide healthier versions of traditional recipes, and might also provide support for food procurement and time management for food preparation and budgeting (Ross and Geil 2010).

Like in other populations (Schlundt et al. 1994; Marcy et al. 2011; Faramand et al. 2015), cravings and time constraints were also mentioned by the participants as barriers to adhering to recommendations. Providing patients with tips on how to eat healthier meals away from home could be helpful.

Given our research group's past experience with menu planning as part of nutrition education for people with T2D (Asaad et al. 2016), we queried whether a Chinese menu plan would benefit participants. Participants showed support and excitement for this idea: "If you could provide Chinese immigrants with a menu plan, I'm in urgent need. I need it very much! I hope you guys can provide this kind of information, guiding us on meal planning" [DAC12] and "... I hope to see the Chinese menu plan. Especially for Chinese people, it will be very useful, because most of us don't eat western food very often. Of course sometimes we do. Like, when we go to banquets, we would also eat western food..." [DAC14]. When asked about the format, participants tended to prefer a more flexible rather than a fixed menu plan: “... The (nutrition) breakdown in details. Once I have that in details, then I can follow... Like, I can incorporate the information I have and then can prepare it with my own menu, right? I really need to know, one cup of cooked white rice, jasmine (rice) for example, one cup of the cooked rice, what is the percentage of carbohydrates and sugar? And then because I know myself, I'm only allowed how many units of carbohydrate. That's why I've started counting since the morning..." [DAC04] and "... you can provide several options. What to eat for breakfast and what to eat for lunch and dinner. You can provide a few options, because if there's only one option and we don't have the ingredients for it, we won't be able to follow it ..." [DAC11], providing multiple options for each meal to accommodate food availability. Other suggestions for a menu plan included: simple and easy to follow, consider the whole family and not just the client with diabetes, inclusive of different types of food including dessert and drinks, based on research evidence, financially accessible, use spices to improve taste, appropriate for both treatment and prevention of T2D, and modify traditional recipes to reduce unhealthy ingredients.

Although acculturation was not purposefully evaluated in the interviews, some elements were reflected therein. The average acculturation was relatively low in the study sample, which was consistent with the following preferences found in interviews: speaking Chinese, reading information written in Chinese, eating Chinese food primarily, and attending the Chinese Community Centre health seminars. Interestingly, while the participants interviewed were concerned about their diet and nutrition, their diet quality was similar and their adherence to nutrition recommendations higher than the mainly Caucasian cohorts previously reported by our group (Asaad et al. 2016; Raj et al. 2018).

A strength of the study is that the sample, although small, had a wide range of age and immigration duration, which makes it more representative of the Chinese immigrant population. Another strength is that the study was designed around a conceptual model (Zeng et al. 2014) that had been developed to explain factors influencing Chinese immigrants' health behaviours. Concurrent data collection and analysis could help determine data saturation, which determines an ideal sample size. However, according to a detailed analysis of qualitative research studies, a sample size of 10-20 is among the most common sample sizes (Mason 2010). Hence, a sample size of 14 is acceptable and many of the conclusions were substantiated by the literature. In qualitative research, documentation of processes to increase validity, reliability, and generalizability is important (Leung 2015). While we followed protocols to increase generalizability and validity, the reliability would have been increased by having another researcher independently code the transcripts. This was not possible without translating all of 
the transcripts into English, which in itself would have increased the potential for misinterpretation. Other limitations included the relatively good health behaviours of the participants (both diet and physical activity), which may have precluded identification of additional barriers.

This study largely validated the Conceptual Model of Diabetes Self-management among Chinese Immigrants (Zeng et al. 2014) although barriers related to traditional health beliefs such as traditional Chinese medicine and yin-yang were not articulated by this group of participants. Culturally relevant education programs could enhance immigrants' knowledge on healthy eating practice and food choices because the staple foods remained traditional among immigrants and most participants showed support to the idea of developing a Chinese menu plan. Lack of culturally relevant information written in Chinese and lack of detailed instructions on meal planning are perceived disadvantages of current dietary recommendations and prevented Chinese immigrants from following recommendations properly. These limitations should be considered in future nutrition programming. Flexible, detailed examples of meal planning was requested by these Chinese immigrants. It is important to find the factors that determine food acceptability, and take these factors into consideration when developing dietary interventions.

\section{Acknowledgements}

The study was funded by the Alberta Diabetes Institute, Alberta Diabetes Foundation and Alberta Health Services. Local grocery stores (Sobeys, Superstore) provided participants' gift cards. FD received a China Scholarship Council stipend. We thank Anran Zhang (AZ) for helping with the recruitment of participants and verification of the transcript coding. The participants are thanked for volunteering to be involved in this study.

\section{Author contributions}

$\mathrm{FD}$ and $\mathrm{CBC}$ conceived and designed the study. FD performed the experiments/collected the data. FD and $\mathrm{CBC}$ analyzed and interpreted the data. $\mathrm{CBC}$ contributed resources. FD and $\mathrm{CBC}$ drafted or revised the manuscript.

\section{Competing interests}

The authors have declared that no competing interests exist.

\section{Data availability statement}

All relevant data are within the paper and in the Supplementary Material.

\section{Supplementary Materials}

The following Supplementary Material is available with the article through the journal website at doi:10.1139/facets-2019-0001.

Supplementary Material 1

\section{References}

Alangh A., Chiu M, and Shah BR. 2013. Rapid increase in diabetes incidence among Chinese Canadians between 1996 and 2005. Diabetes Care, 36(10): 3015-3017. PMID: 23723356 DOI: $10.2337 / \mathrm{dc} 13-0052$

Anderson J, Wiggins S, and Rajwani R. 1995. Living with a chronic illness: Chinese-Canadian and Euro-Canadian women with diabetes-exploring factors that influence management. Social Science and Medicine, 41: 181-195. PMID: 7667681 DOI: 10.1016/0277-9536(94)00324-M 
Asaad G, Sadegian M, Lau R, Xu Y, Soria-Contreras DC, Bell RC,et al. 2015. The reliability and validity of the perceived dietary adherence questionnaire for people with type 2 diabetes. Nutrients, 7: 5484-5496. PMID: 26198247 DOI: 10.3390/nu7075231

Asaad G, Soria-Contreras DC, Bell RC, and Chan CB. 2016. Effectiveness of a lifestyle intervention in patients with type 2 diabetes: the Physical Activity and Nutrition for Diabetes in Alberta (PANDA) trial. Healthcare, 4: 73. PMID: 27690122 DOI: 10.3390/healthcare4040073

Bader A, Gougeon R, Joseph L, Da Costa D, and Dasgupta K. 2013. Nutritional education through internet-delivered menu plans among adults with type 2 diabetes mellitus: pilot study. JMIR Research Protocols, 2: e41-e46. PMID: 24185033 DOI: 10.2196/resprot.2525

Beasley JM, Yi SS, Ahn J, Kwon SC, and Wylie-Rosett J. 2018. Dietary patterns in Chinese Americans are associated with cardiovascular disease risk factors, the Chinese American Cardiovascular Health Assessment (CHA CHA). Journal of Immigrant Minority Health: 1-9 (published online 28 August 2018). PMID: 30097786 DOI: $10.1007 /$ s10903-018-0800-z

Cao K, He Y, and Yang X. 2014. The association between eating out of home and overweight/obesity among Chinese adults. Chinese Journal of Preventive Medicine, 48: 1088-1092. PMID: 25619222

Chui T, Tran K, and Flanders J. 2005. Chinese Canadians: enriching the cultural mosaic. Statistics Canada, Ottawa, Ontario. Catalogue No. 11-008: 24-32.

Contento IR. 2011. Nutrition education: linking research, theory and practice. Jones and Bartlett Learning, Burlington, Vermont.

Coyne T, Olson M, Bradham K, Garcon M, Gregory P, and Scherch L. 1995. Dietary satisfaction correlated with adherence in the modification of diet in renal disease study. Journal of the American Dietetics Association, 95(11): 1301-1306. PMID: 7594127 DOI: 10.1016/S0002-8223(95)00341-X

Creswell JW. 2007. Qualitative inquiry and research design. Sage Publications, Thousand Oaks, California.

Cunningham C, Johnson S, Cowell B, Soroudi N, Isaacson S, Davis N, et al. 2006. Menu plans in a diabetes self-management weight loss program. Journal of Nutrition Education and Behavior, 38: 264-266. PMID: 16785099 DOI: 10.1016/j.jneb.2006.01.013

Deng F, Zhang A, and Chan CB. 2013. Acculturation, dietary acceptability, and diabetes management among Chinese in North America. Frontiers Endocrinology (Lausanne), 4: 108. PMID: 23986745 DOI: $10.3389 /$ fendo.2013.00108

Deng F, Zhang A,and Chan CB. 2018. Nutrition interventions for type 2 diabetes in Chinese populations: a scoping review. Journal of Immigrant Minority Health: 1-16 (published online 6 December 2018). PMID: 30515634 DOI: 10.1007/s10903-018-0845-Z

Faramand M, Amiri P, Tehrani F, Momenan AA, Mirmiran P, and Azizi F. 2015. What are the main barriers to healthy eating among families? A qualitative exploration of perceptions and experiences of Tehranian men. Appetite, 89: 291-297. PMID: 25725485 DOI: 10.1016/j.appet.2015.02.025

Franzen L, and Smith C. 2009. Acculturation and environmental change impacts dietary habits among adult Hmong. Appetite, 52: 173-183. PMID: 18848592 DOI: 10.1016/j.appet.2008.09.012

Garriguet D. 2009. Diet quality in Canada. Health Reports, 20: 41-42. PMID: 19813438 
Godin G. 2011. The Godin-Shephard leisure-time physical activity questionnaire. The Health and Fitness Journal of Canada, 4: 18-22.

Gonzalez-Zacarias AA, Mavarez-Martinez A, Arias-Morales CE, Stoicea N, and Rogers B. 2016. Impact of demographic, socioeconomic, and psychological factors on glycemic self-management in adults with type 2 diabetes mellitus. Frontiers Public Health, 12: 195. PMID: 27672634 DOI: 10.3389/fpubh.2016.00195

Hyman I, Shakya Y, Jembere N, Gucciardi E, and Vissandjee B. 2017. Provider- and patient-related determinants of diabetes self-management among recent immigrants: implications for systemic change. Canadian Family Physician, 63: e137-e144. PMID: 28209706

Income Statistics Division. 2015. Low income lines, 2013-2014. Statistics Canada: Ottawa, Ontario.

Jimenez-Cruz A, Bacardi-Gascon M, Turnbull WH, Rosales-Garay P, and Severino-Lugo I. 2003. A flexible, low-glycemic index Mexican-style diet in overweight and obese subjects with type 2 diabetes improves metabolic parameters during a 6-week treatment period. Diabetes Care, 26(7): 1967-1970. PMID: 12832297 DOI: 10.2337/diacare.26.7.1967

Kandula NR, Diez-Roux AV, Chan C, Daviglus ML, Jackson SA, Ni H, et al. 2008. Association of acculturation levels and prevalence of diabetes in the multi-ethnic study of atherosclerosis (MESA). Diabetes Care, 31(8): 1621-1628. PMID: 18458142 DOI: 10.2337/dc07-2182

Kwok S, Mann L, Wong K, and Blum I. 2009. Dietary habits and health beliefs of Chinese Canadians. Canadian Journal of Dietetic Practice and Research, 70: 73-80. PMID: 19515270 DOI: $10.3148 /$ 70.2 .2009 .73

Kwon YS, and Ju SY. 2014. Trends in nutrient intakes and consumption while eating-out among Korean adults based on Korea National Health and Nutrition Examination Survey (1998-2012) data. Nutrition Research and Practice, 8(6): 670-678. PMID: 25489407 DOI: 10.4162/nrp.2014.8.6.670

Lear SA, Humphries KH, Hage-Moussa S, Chockalingam A, and Mancini GB. 2009. Immigration presents a potential increased risk for atherosclerosis. Atherosclerosis, 205: 584-589. PMID: 19181317 DOI: 10.1016/j.atherosclerosis.2008.12.037

Leung L. 2015. Validity, reliability, and generalizability in qualitative research. Journal Family Medicine Primary Care, 4(3): 324-327. PMID: 26288766 DOI: 10.4103/2249-4863.161306

Lv N, and Brown JL. 2010. Chinese American family food systems: impact of Western influences. Journal of Nutrition Education and Behavior, 42(2): 106-114. PMID: 20219723 DOI: $10.1016 /$ j.jneb.2009.04.005

Lv N, and Cason KL. 2004. Dietary pattern change and acculturation of Chinese Americans in Pennsylvania. Journal of the American Dietetic Association, 104(5): 771-778. PMID: 15127063 DOI: 10.1016/j.jada.2004.02.032

Marcy TR, Britton ML, and Harrison D. 2011. Identification of barriers to appropriate dietary behavior in low-income patients with type 2 diabetes mellitus. Diabetes Therapy, 2: 9-19. PMID: 22127765 DOI: $10.1007 / \mathrm{s} 13300-010-0012-6$

Marshall C, and Rossman GB. 2006. Designing qualitative research. Sage Publications, Thousand Oaks, California. 
Mason MS. 2010. Sample size and saturation in PhD studies using qualitative interviews. Forum Qualitative Sozialforschung/Forum: Qualitative Social Research, 11(3). DOI: 10.17169/fqs-11.3.1428

Merriam SB. 2002. Qualitative research in practice: examples for discussion and analysis. Jossey-Bass, San Francisco, California.

Moreira T, Severo M, Oliveira A, Ramos E, Rodrigues S, and Lopes C. 2015. Eating out of home and dietary adequacy in preschool children. British Journal of Nutrition, 114: 297-305. PMID: 26082269 DOI: $10.1017 /$ S0007114515001713

Pan YL, Dixon Z, Himburg S, and Huffman F. 1999. Asian students change their eating patterns after living in the United States. Journal of the American Dietetic Association, 99(1): 54-57. PMID: 9917732 DOI: 10.1016/S0002-8223(99)00016-4

Perez CE. 2002. Health status and health behaviour among immigrants. Supplement to health reports. Statistics Canada, Ottawa, Ontario. Vol. 13, pp. 1-12.

Pomerleau J., Lock K, Knai C, and McKee M. 2005. Interventions designed to increase adult fruit and vegetable intake can be effective: a systematic review of the literature. Journal of Nutrition, 135: 2486-2495. PMID: 16177217 DOI: 10.1093/jn/135.10.2486

Raj GD, Hashemi Z, Soria Contreras DC, Babwik S, Maxwell D, Bell RC, et al. 2018. Adherence to diabetes dietary guidelines assessed using a validated questionnaire predicts glucose control in adults with type 2 diabetes. Canadian Journal of Diabetes, 42(1): 78-87. PMID: 28648765 DOI: $10.1016 / j . j c j d .2017 .04 .006$

Rosenmoller DL, Gasevic D, Seidell J, and Lear SA. 2011. Determinants of changes in dietary patterns among Chinese immigrants: a cross-sectional analysis. The International Journal of Behavior Nutrition and Physical Activity, 8: 42. PMID: 21592378 DOI: 10.1186/1479-5868-8-42

Ross T, and Geil P. 2010. Healthy eating on a lean budget: diabetes meals for less. Diabetes Spectrum, 23: $120-123$. DOI: 10.2337 /diaspect.23.2.120

Sanou D, O'Reilly E, Ngnie-Teta I, Batal M, Mondain N, Andrew C, et al. 2014. Acculturation and nutritional health of immigrants in Canada: a scoping review. Journal of Immigrant Minority Health, 16(1): 24-34. PMID: 23595263 DOI: 10.1007/s10903-013-9823-7

Satia JA, Patterson RE, Taylor VM, Cheney CL, Shiu-Thornton S, Chitnarong K, et al. 2000. Use of qualitative methods to study diet, acculturation, and health in Chinese-American women. Journal of the American Dietetic Association, 100(8): 934-940. PMID: 10955052 DOI: $10.1016 /$ S0002-8223(00)00269-8

Schlundt DG, Rea MR, Kline SS, and Pichert JW. 1994. Situational obstacles to dietary adherence for adults with diabetes. Journal of the American Dietetic Association, 94(8): 874-879. PMID: 8046181 DOI: $10.1016 / 0002-8223(94) 92367-1$

Soria-Contreras DC, Bell RC, McCargar LJ, and Chan CB. 2014. Feasibility and efficacy of menu planning combined with individual counselling to improve health outcomes and dietary adherence in people with type 2 diabetes: a pilot study. Canadian Journal of Diabetes, 38: 320-325. PMID: 25175312 DOI: 10.1016/j.jcjd.2014.03.009

Statistics Canada. 2012. Lunar New Year... by the numbers. The Daily [online]: Available from www42.statcan.gc.ca/smr08/2012/smr08_161_2012-eng.htm. 
Suinn RM, Ahuna C, and Khoo G. 1992. The Suinn-Lew Asian Self-Identity Acculturation Scale: concurrent and factorial validation. Educational and Psychological Measurement, 52: 1041-1046. DOI: $10.1177 / 0013164492052004028$

Tan C-B. 2011. Chinese food and foodways in Southeast Asia and beyond. NUS Press Pte Ltd, Singapore, Singapore.

Thabit H, Shah SD, Nash M, Brema I, Nolan JJ, and Martin G. 2009. Globalization, immigration and diabetes self-management: an empirical study amongst immigrants with type 2 diabetes mellitus in Ireland. Quarterly Journal of Medicine, 102: 713-720. PMID: 19736289 DOI: 10.1093/gjmed/hcp 113

Walks RA, and Bourne LS. 2006. Ghettos in Canada's cities? Racial segregation, ethnic enclaves and poverty concentration in Canadian urban areas. The Canadian Geographer, 50(3): 273-297. DOI: $10.1111 / j .1541-0064.2006 .00142 . x$

Wight D, Wimbush E, Jepson R, and Doi L. 2016. Six steps in quality intervention development (6SQuID). Journal of Epidemiology and Community Health, 70: 520-525. PMID: 26573236 DOI: $10.1136 /$ jech-2015-205952

Xu Y., Pan W, and Liu H. 2011. The role of acculturation in diabetes self-management among Chinese Americans with type 2 diabetes. Diabetes Research and Clinical Practice, 93: 363-370. PMID: 21636157 DOI: 10.1016/j.diabres.2011.05.010

Zeng B, Sun W, Gary RA, Li C, and Liu T. 2014. Towards a conceptual model of diabetes selfmanagement among Chinese immigrants in the United States. International Journal of Environmental Research and Public Health, 11(7): 6727-6742. PMID: 24978878 DOI: 10.3390/ijerph110706727 